\title{
PHOTOVISUAL MAGNITUDE DIFFERENCES \\ OF DOUBLE STARS
}

\author{
K. Aa. STR A N D \\ U.S. Naval Observatory, Washington, D.C., U.S.A.
}

\begin{abstract}
Differences in magnitudes between the components of 874 double stars have been derived from visual estimates on several series of multi-exposure photographic plates. The plates were obtained with long focus refractors primarily for the purpose of determining the relative positions of double stars according to the well-known Hertzsprung method.

Except for binaries with magnitude differences less than half a magnitude, plates were taken with objective gratings limiting the effective magnitude differences between the components to half a magnitude.

Similar to what has been done in variable star work, it was found possible to estimate the magnitude difference to the nearest tenth from this material by visual inspection, using a seven power eyepiece.

The internal mean error of a single estimate of a magnitude is $\pm 0^{m} .064$, while the external mean error of a single value in the catalogue based upon an average of three independent determinations is $\pm 0^{m} .054$.

Comparisons with other photometric series of this type have been made. In particular the photoelectric series by Eggen $(1963,1966)$ and by Johnson $(1953)$ show close agreements, with no systematic differences.

The results will appear in Publ. U.S. Naval Obs., 2nd Series, 18, Pt. V.
\end{abstract}

\section{References}

Eggen, O. J.: 1963, Astron. J. 68, 484.

Eggen, O. J.: 1966, Roy. Obs. Bull. Serie E, No. 120.

Johnson, H. L.: 1953, Astrophys. J. 117, 361.

\section{Discussion}

Franz emphasizes that the extensive plate series available for a number of double stars are an important potential source of information on variable double-star components. For instance, he has reasons to assume that $\gamma$ Leo might be variable. His observations, though not numerous, show thus far a large dispersion of almost 0.2 mag.

Strand replies that he believes to have found two or three new variable stars. Nothing peculiar was noticed about $\gamma$ Leo. However, estimates of $\Delta m$ for each star were generally made on four or five plates at most. 